See Article page XXX.

\section{Commentary: Seeing through the fog: Longitudinal strain as a predictor of survival after septal myectomy}

\author{
Charles M. Wojnarski, MD, MS, and \\ Robert J. Moraca, MD
}

Cui and colleagues ${ }^{1}$ present a retrospective analysis of left ventricular longitudinal strain pattern (as it relates to survival) in a large cohort of patients undergoing septal myectomy for obstructive hypertrophic cardiomyopathy (HCM) with preserved left ventricular ejection fraction (LVEF). ${ }^{1}$ From 2007 to 2016, 857 patients underwent septal myectomy for obstructive HCM at the Mayo Clinic and had available preoperative transthoracic echocardiography with longitudinal strain measurements. The authors employed a Cox proportional hazard model to identify and adjust for covariates associated with survival and found global longitudinal strain (GLS) to be a strong predictor of long-term survival after septal myectomy for obstructive HCM (hazard ratio [ $-1 \%$ GLS increment], $0.89 ; 95 \%$ confidence interval, $0.83-0.96 ; P=.003$ ). They also performed a subgroup analysis of 64 patients with postoperative GLS measurements and compared strain patterns before and after septal myectomy.

The group at Mayo Clinic has been incrementally adding to our knowledge of obstructive HCM and outcomes after septal myectomy for decades. This current report provides an additional prognostic variable among HCM patients treated with surgery.

Long-term outcomes of patients with HCM and depressed LVEF $(<50 \%)$ have been shown to be worse than those with preserved function. ${ }^{2}$ However, given the physiologically

\footnotetext{
From the Section of Cardiac Surgery, Department of Surgery, Virginia Mason Medical Center, Seattle, Wash.

Disclosures: The authors reported no conflicts of interest.

The Journal policy requires editors and reviewers to disclose conflicts of interest and to decline handling or reviewing manuscripts for which they may have a conflict of interest. The editors and reviewers of this article have no conflicts of interest.

Received for publication Sept 28, 2021; revisions received Sept 28, 2021; accepted for publication Sept 29, 2021.

Address for reprints: Charles M. Wojnarski, MD, MS, Section of Cardiac Surgery, Department of Surgery, Virginia Mason Medical Center, 1100 Ninth Ave, X3CAR, Seattle, WA 98101 (E-mail: charles.wojnarski@virginiamason.org).

J Thorac Cardiovasc Surg 2021; $\mathbf{\square}: 1-2$

$0022-5223 / \$ 36.00$

Copyright (C) 2021 by The American Association for Thoracic Surgery

https://doi.org/10.1016/j.jtcvs.2021.09.056
}

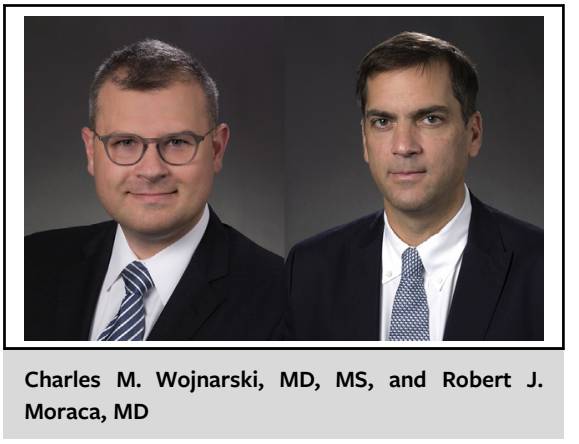

CENTRAL MESSAGE

Reduced global longitudinal strain in patients with obstructive hypertrophic cardiomyopathy predicts lower survival and may help identify patients who would benefit from early septal myectomy.

hyperdynamic state of the left ventricle in HCM, LVEF has been a poor predictor of outcomes after septal myectomy in patients without end-stage disease. In our view, the 2 most important findings of this article are that GLS, even after multivariable adjustment, predicted survival and that after septal myectomy, regional strains were more uniform and evenly distributed than preoperatively.

It is important to note that this cohort only included those undergoing septal myectomy and may not be representative of all patients with HCM. However, earlier work from the Cleveland Clinic, including surgically treated and medically managed patients, reached similar conclusionsfinding that worsening of GLS over time was associated with reduced survival in medically managed patients and septal myectomy was associated with improved survival. ${ }^{3}$

Because septal myectomy has become a safe, predictable treatment for patients with obstructive HCM, it is important to understand when intervention should be undertaken even in those with minimal symptoms. This report suggests that using longitudinal strain patterns, in addition to traditional indications for intervention, might serve to inform timing of intervention; however, more study is needed before any firm recommendations can be made.

\section{References}

1. Cui H, Schaff HV, Nishimura RA, Geske JB, Dearani JA, Newman DB, et al. Preoperative Left ventricular longitudinal strain predicts outcome of septal myectomy 
for obstructive hypertrophic cardiomyopathy. J Thorac Cardiovasc Surg. 2021. $\mathrm{XX}: \mathrm{XX}-\mathrm{X}$.

2. Marstrand P, Han L, Day SM, Olivotto I, Ashley EA, Michels M, et al. Hypertrophic cardiomyopathy with left ventricular systolic dysfunction: insights from the SHaRe registry. Circulation. 2020;141:1371-83.
3. Tower-Rader A, Betancor J, Popovic ZB, Sato K, Thamilarasan M, Smedira NG, et al. Incremental prognostic utility of left ventricular global longitudinal strain in hypertrophic obstructive cardiomyopathy patients and preserved left ventricular ejection fraction. J Am Heart Assoc. 2017;6: e006514. 